\title{
Beyond synchronization: String instability in coupled harmonic oscillator systems
}

\author{
Bo Yu ${ }^{1}$, James S. Freudenberg ${ }^{2, *, \dagger}$, R. Brent Gillespie ${ }^{1}$ and Richard H. Middleton ${ }^{3}$ \\ ${ }^{1}$ Department of Mechanical Engineering, University of Michigan, Ann Arbor, MI 48109-2125, USA \\ ${ }^{2}$ Department of Electrical Engineering and Computer Science, University of Michigan, Ann Arbor, MI 48109-2122, USA \\ ${ }^{3}$ School of Electrical Engineering and Computer Science, University of Newcastle, Newcastle, Australia
}

\begin{abstract}
SUMMARY
In this paper, we consider the problem of disturbance response and error amplification for a simple system of coupled harmonic oscillators. We first suppose that identical oscillators are connected in a string in which each oscillator attempts to track its predecessor by using the same control law that depends on the relative position information from its immediate predecessor. Such an oscillator string is called a homogeneous oscillator string with predecessor-following architecture. Motivated by terminology from the problem of vehicle platooning, we say that the synchronized oscillator system is string unstable if the effect of a disturbance to the lead oscillator is amplified as it propagates along the string. With the use of a new Bode-like integral relation that must be satisfied by the complementary sensitivity function, we provide sufficient conditions for string instability. The sufficient conditions show that any string of oscillators that satisfies certain time domain performance specifications and bandwidth limitations must necessarily be string unstable. We further introduce a concept of time headway for the oscillator system and extend our analysis of string instability to consider the heterogeneous oscillator string and a more general communication range. Copyright $(C$ 2014 John Wiley \& Sons, Ltd.
\end{abstract}

Received 21 January 2014; Revised 27 May 2014; Accepted 4 July 2014

KEY WORDS: harmonic oscillators; string instability; error amplification; consensus; synchronization

\section{INTRODUCTION}

Many researchers have studied the problem of synchronization in systems of coupled oscillators. As noted in [1-3], this problem may be viewed as a special case of consensus control in multi-agent systems, in which each oscillator communicates with a subset of its neighbors for the purpose of achieving synchronization. The synchronization of oscillators finds applications in many different areas [4-6], for example, synchronously flashing fireflies, microwave oscillations, and electrical power networks. Depending on the communication topology, the oscillators may or may not be able to achieve synchronization. The ability to do so also depends on the presence of communication time delays and changes in the communication topology. In the present paper, we study the effect of a disturbance on a system of coupled oscillators. Specifically, we wish to know whether the effect of a disturbance to one oscillator will be amplified or diminished as it propagates through the synchronized oscillator system.

Our approach to the problem of disturbance propagation for a system of oscillators is inspired by the literature on the problem of string instability that may arise in vehicle platooning (e.g. [7-13]). Specifically, we first consider a string of oscillators, in which one is the leader, and with which the

\footnotetext{
*Correspondence to: James S. Freudenberg, Department of Electrical Engineering and Computer Science, University of Michigan, Ann Arbor, MI 48109-2122 USA.

${ }^{\dagger}$ E-mail: jfr@umich.edu
} 
remainder attempts to synchronize their oscillations by tracking only their immediate predecessor in the string. It is known that this predecessor-following strategy will exhibit string instability under certain conditions for vehicle platoons. More complex communication schemes, on the other hand, may allow the design of control laws that are string stable. For example, when each vehicle may communicate with both its immediate predecessor and successor, a controller supporting string stability exists. Early studies of string instability were undertaken in the context of specific control laws, such as PID [8]. This made a comparison between different communication schemes problematic, in that the observed string instability may have been due to a poor choice of controller gains rather than the communication scheme adopted. The authors in [10], on the other hand, show that, under appropriate hypotheses, certain communication topologies will lead to string instability for any linear controller. To show this, they applied the theory of fundamental design limitations [14], which enables such general statements to be made assuming only that the controller is stabilizing. In [10], it is assumed that all the vehicles have the same model and use the same control law, and it is shown that the predecessor-following control law will necessarily lead to problems of string instability for constant spacing between vehicles. The authors of [11] greatly extend the results in [10] by considering heterogeneous platoons and more general spacing policies and communication topologies. However, this string instability analysis in vehicle platooning cannot directly be used to study the disturbance response and error propagation problems in synchronized oscillator systems mainly due to the fact that vehicles are modeled by integrators with one or two poles at origin and harmonic oscillators' model has two purely imaginary poles.

Many papers on oscillator synchronization use the first order, nonlinear Kuramoto model [5], or an appropriate extension thereof $[1,2]$. Other famous oscillator systems in nonlinear physics and chaos include Toda chain [15] and Fermi-Pasta-Ulam models [16]. In order to apply the theory of fundamental design limitations, we instead use the second order, linear oscillator model described in [17]. This will enable us to use the fact that such oscillators have poles on the imaginary axis, and to generalize the results from the theory of fundamental limitations that were used in $[10,11]$. We start our analysis by studying the problem of string instability in a string of identical harmonic oscillators, each trying to track its immediate predecessor using an identical control law corresponding to the predecessor-following strategy used in vehicle platooning studies. By applying the theory of fundamental design limitations [14], we develop a Bode-like integral relation that holds for any stabilizing control law. This integral relation may be used to show that any string of oscillators that satisfies certain time domain performance specifications, bandwidth limitations, and communication delays must necessarily be string unstable.

String instability is clearly not a desirable feature in a string of oscillators. We therefore study strategies that may enable string stability to be present. These are motivated by similar studies of vehicle platooning that include the use of heterogeneous control laws, an extended communication range, and time headway $[10,11]$. In this paper, we develop an extension of the concept of time headway that is applicable to oscillator systems, and show that including such headway in the control law can also result in string stability. We then extend our string instability analysis to consider the heterogeneous controller design and a more general communication range between oscillators. Preliminary versions of this paper appear in two conference publications $[18,19]$.

The remainder of this paper is outlined as follows. In Section 2, we provide some background on oscillator synchronization and review the integral constraint on the complementary sensitivity function that was used in [10] to study the string instability problem in vehicle platooning. This integral constraint is not applicable to our problem, and thus in Section 3, we propose a more general integral relation that may be applied to oscillator systems. We use this result to derive three sufficient conditions for string instability in Section 4. Specifically, we assume that a controller has been designed that satisfies certain time and frequency domain design specifications, and show that this assumption implies a lower bound on the peak in magnitude response of the complementary sensitivity function; if this lower bound exceeds one, then string instability is present. We then introduce the time headway concept for the oscillator system to improve the string stability and extend our string instability analysis to consider heterogeneous strings and a more general communication range in Section 5. The results of the paper are illustrated with numerical examples in Section 6. Conclusions and future research directions are given in Section 7. 
Notation: OLHP, CLHP, ORHP, and CRHP denote the open-left, closed-left, open-right, and closed-right halves of the complex plane, respectively. We use Re and Im to represent the real and imaginary parts of a complex number, respectively. We use log to denote the natural logarithm and arg to denote the principal branch of the argument of a complex number. The relative degree $r$ of a rational transfer function is the degree of its denominator minus the degree of its numerator polynomial. The notation $P(s) \star u(t)$ is used to denote the time response with zero initial conditions of a linear time-invariant system with transfer function $P(s)$ and input $u(t)$. The notation $\lceil x\rceil$ represents the smallest integer no smaller than $x$. The product notation that includes matrices is defined as follows: $\prod_{i=1}^{n} M_{i} \triangleq M_{n} M_{n-1} \cdots M_{2} M_{1}$.

\section{PRELIMINARY RESULTS ON SYNCHRONIZATION OF OSCILLATORS AND STRING INSTABILITY}

The objective of synchronization is to find the conditions on network topology and coupling algorithms that guarantee that the oscillators can collectively achieve synchronized behaviors. The existing literature shows that the ability to achieve synchronization in oscillator systems depends on both the communication topology and the control algorithms that prescribe how one oscillator interacts with its neighbors [17]. Even if an oscillator system can achieve synchronization, other issues such as disturbance response will affect its performance and practicality. In the following, we will demonstrate the problem of disturbance response and error amplification in a synchronized homogeneous oscillator system with a simple communication topology.

Consider the series connection, or string, of $n$ single-loop feedback systems depicted in Figure 1. We assume that these systems are all identical, with each plant described by a proper rational transfer function of the form

$$
P(s)=P_{0}(s) \frac{1}{s^{2}+\alpha^{2}},
$$

where $P_{0}(s)$ has no zeros at $s= \pm j \alpha$, and with rational and stabilizing controller $C(s)$. Each plant thus contains the dynamics of a harmonic oscillator with natural frequency $\alpha$ radians/second.

Suppose that we desire each oscillator in the string to track the position of its immediate predecessor. Following the terminology used in vehicle platooning, we refer to the system in Figure 1 as a predecessor-following control architecture. Denote the commanded position to the lead oscillator by $r_{1}(t)$, and the positions and tracking errors of the $i$ th oscillator as $y_{i}(t)$ and $e_{i}(t)$, respectively. Let $d_{\text {out }}(t)$ denote a disturbance entering at the output of the first oscillator. Each error signal can thus be expressed as

$$
e_{i}(t)=\left\{\begin{array}{c}
r_{1}(t)-y_{1}(t), \quad i=1, \\
y_{i-1}(t)-y_{i}(t), i \geqslant 2 .
\end{array}\right.
$$

Define the open loop transfer function $L(s)=P(s) C(s)$, and the sensitivity and complementary sensitivity functions by

$$
S(s)=\frac{1}{1+L(s)}, \quad T(s)=\frac{L(s)}{1+L(s)},
$$

respectively. Then the Laplace transforms of the tracking error signals satisfy

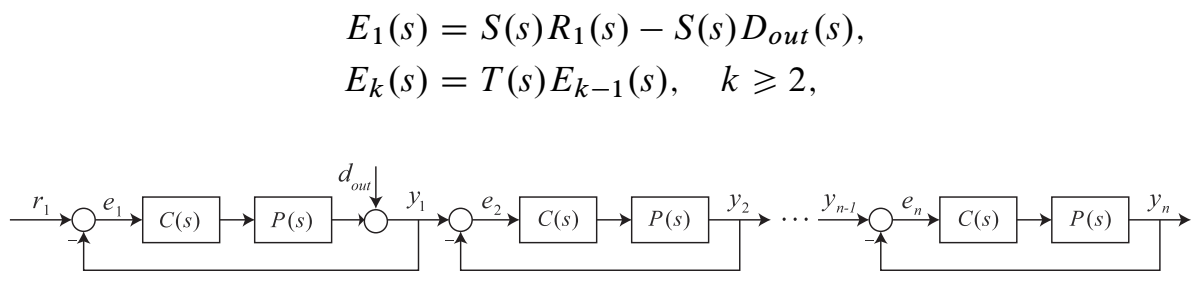

Figure 1. Block diagram depicting a string of stabilized oscillators with length $n$. 
and thus

$$
E_{k}(s)=T^{k-1}(s) E_{1}(s), \quad k \geqslant 1 .
$$

The presence of the plant poles at $\pm j \alpha$ implies that $T( \pm j \alpha)=1$ and $S( \pm j \alpha)=0$. Hence, the steady state error (SSE) in response to an input of the form $r_{1}(t)=A \sin (\alpha t+\phi)$ will be equal to zero for each oscillator in the string. In this way, the motion of all the oscillators in the string will synchronize to that of the lead oscillator. We see from (4) that the command $r_{1}(t)$ and output disturbance $d_{\text {out }}(t)$ affect the system symmetrically, and thus conclusions drawn about the command response also apply to the disturbance response.

In such a homogeneous oscillator system, the synchronization problem reduces to the design of a controller $C(s)$ such that $T(s)$ is stable. Suppose $T(s)$ is stable and there exists a frequency $\omega$ for which $|T(j \omega)|>1$. Then (5) implies that any disturbance to the lead oscillator at this frequency will be amplified as it propagates to successive oscillators. As the number of oscillators increases, the error will be amplified without bound, and the string in Figure 1 will be string unstable.

Similar phenomena of string instability have appeared in vehicle platooning. One approach to study the string instability problem in vehicle platooning is to use the theory of fundamental limitations [14] to derive conditions for string instability that apply to all linear time-invariant controllers. In studies of string instability in vehicle platooning, one may derive sufficient conditions for string instability using the following integral relation, dual to the Bode sensitivity integral, that must be satisfied by the complementary sensitivity function [14, Theorem 3.1.5].

\section{Theorem 1}

(a) Consider a unity feedback system with plant $P(s)$ and stabilizing controller $C(s)$. Assume that $L(s)$ is rational and proper, with $N_{z}$ zeros in the ORHP, $\left\{z_{i}: i=1, \ldots, N_{z}\right\}$. Assume further that $L(s)$ may be factored as $L(s)=L_{0}(s) / s^{k}$, where $k \geqslant 1$ and $L_{0}(s)$ has neither poles nor zeros at $s=0$. Then

$$
\int_{0}^{\infty} \log |T(j \omega)| \frac{d \omega}{\omega^{2}}=\frac{\pi}{2} T^{\prime}(0)+\pi \sum_{i=1}^{N_{z}} \frac{1}{z_{i}},
$$

where $T^{\prime}(0)=\lim _{s \rightarrow 0} d T(s) / d s$.

(b) Suppose, in addition, that $k \geqslant 2$. Then $T^{\prime}(0)=0$, and

$$
\int_{0}^{\infty} \log |T(j \omega)| \frac{d \omega}{\omega^{2}}=\pi \sum_{i=1}^{N_{z}} \frac{1}{z_{i}} .
$$

Because complex zeros must occur in conjugate pairs, it follows that the right-hand side of (7) is real and nonnegative. It follows immediately from (7) that if $L(s)$ has a double integrator, then necessarily there must exist a frequency for which $|T(j \omega)|>1$. This fact was used in [10] to show that a platoon of identical vehicles in the predecessor-following control architecture must be string unstable. Recently, the results of [10] were generalized in [11] to provide sufficient conditions for string instability with heterogeneous platoons and more general control architectures. The assumption of a double integrator is reasonable for the study of vehicle platoons to model the vehicle with torque as input and position as output. If only a single integrator is present, then an integral constraint still holds, but need not imply that $|T(j \omega)|>1$ because of the term $T^{\prime}(0)$, which may be negative. As discussed in [14], this term is inversely proportional to the velocity constant of a Type 1 feedback system.

Theorem 1 is not applicable to our study of oscillators because it is based on the double integrator model, and the harmonic oscillator has a pair of pure imaginary poles. In the following section, we will derive a new generalized complementary sensitivity integral, which can be used for harmonic oscillator systems and includes the integrals in Theorem 1 as special cases. 


\section{A NEW GENERALIZED COMPLEMENTARY SENSITIVITY INTEGRAL}

We propose a new integral relation that the complementary sensitivity function must satisfy whenever $L(s)$ contains a pair of poles on the imaginary axis.

\section{Theorem 2}

Consider a feedback system with plant $P(s)$ given by (1), and stabilizing controller $C(s)$. Suppose that $L(s)$ has $N_{z}$ ORHP zeros $\left\{z_{i}: i=1, \ldots, N_{z}\right\}$ and may be factored as

$$
L(s)=e^{-s \tau} L_{0}(s) \frac{1}{\left(s^{2}+\alpha^{2}\right)^{k}},
$$

where $k \geqslant 1, L_{0}(s) \frac{1}{\left(s^{2}+\alpha^{2}\right)^{k}}$ is proper, $L_{0}(s)$ is rational with no zeros at $s= \pm j \alpha$, and $\tau \geqslant 0$. Then

$$
\int_{0}^{\infty} \log |T(j \omega)| W(\omega, \alpha) d \omega=\frac{\pi}{2} \operatorname{Re}\left(K_{\alpha}\right)+\pi \sum_{i=1}^{N_{z}}\left(\frac{z_{i}}{z_{i}^{2}+\alpha^{2}}\right)+\frac{\pi}{2} \tau,
$$

where

$$
K_{\alpha} \triangleq \lim _{s \rightarrow j \alpha} \frac{d T(s)}{d s}
$$

and the weighting function $W(\omega, \alpha)$ is defined as

$$
W(\omega, \alpha)=\frac{\omega^{2}+\alpha^{2}}{\left(\omega^{2}-\alpha^{2}\right)^{2}} .
$$

Suppose, in addition, that $k \geqslant 2$ and $\tau=0$. Then $K_{\alpha}=0$, and

$$
\int_{0}^{\infty} \log |T(j \omega)| W(\omega, \alpha) d \omega=\pi \sum_{i=1}^{N_{z}}\left(\frac{z_{i}}{z_{i}^{2}+\alpha^{2}}\right) .
$$

Proof

See the Appendix.

In the following section, we will use the integrals in Theorem 2 to derive three sufficient conditions for string instability for a string of oscillators in Section 2. Also, it is worth noting that when $\alpha$ is 0 , the integrals in Theorem 2 reduce to those in Theorem 1 .

\section{HOMOGENEOUS OSCILLATOR STRING}

An immediate result of Theorem 2 is the following sufficient condition for string instability for the homogeneous oscillator system in Section 2.

\section{Theorem 3}

Consider the series connection of feedback systems in Figure 1, with plant (1) and stabilizing compensator $C(s)$. If $L(s)$ has at least $t w o$ pairs of poles at $\pm j \alpha$, then the string of oscillators in Figure 1 is string unstable.

\section{Proof}

Note that the right-hand side of (12) is nonnegative, the time delay term $\frac{\pi}{2} \tau$ is nonnegative, and that $W(\omega, \alpha)>0$ for all frequencies except $\omega=\alpha$. It follows that if $L(s)$ has at least $t w o$ pairs of poles at $\pm j \alpha$, then $K_{\alpha}=0$. Hence, there must exist a frequency for which $|T(j \omega)|>1$, and the string of oscillators in Figure 1 is string unstable. 
Suppose that $L(s)$ contains only a single pair of poles at $\pm j \alpha$, namely, those due to the plant (1). Then $K_{\alpha}$ defined in (10) may be negative and, as a consequence, $|T(j \omega)|$ may be less than one at all frequencies and string instability may not be present.

Recall that the term corresponding to $K_{\alpha}$ in Theorem 1 is inversely proportional to the velocity constant that describes the SSE of a Type 1 feedback system in response to a ramp input. The following result provides a corresponding interpretation for $K_{\alpha}$ and shows that it describes the SSE in response to an input of the form $r_{1}(t)=t \sin \alpha t$.

\section{Theorem 4 (Interpretation of $K_{\alpha}$ )}

(a) Consider the series connection of feedback systems in Figure 1, with plant (1) and stabilizing compensator $C(s)$. Assume that $r_{1}(t)=t \sin \alpha t$, and define the SSE for the first system as the response that persists after the transient response decays, denoted by $e_{1}^{s S}(t)$. Then

$$
e_{1}^{s s}(t)=\left|K_{\alpha}\right| \sin \left(\alpha t+\arg \left(-K_{\alpha}\right)\right) .
$$

(b) Suppose in addition that $\arg \left(-K_{\alpha}\right)=0$. Then in steady state $y_{1}(t)$, is in phase with $r_{1}(t)$, and the steady state response $y_{1}^{s s}(t)$ is given by

$$
y_{1}^{s s}(t)=\left(t-\left|K_{\alpha}\right|\right) \sin \alpha t .
$$

Proof

(a) The Laplace transform of $t \sin (\alpha t)$ is given by $2 \alpha s /\left(s^{2}+\alpha^{2}\right)^{2}$. This fact, together with (4), yields

$$
E_{1}(s)=(1-T(s)) \frac{2 \alpha s}{\left(s^{2}+\alpha^{2}\right)^{2}} .
$$

Noting that $1-T(s)$ has zeros at $\pm j \alpha$, it follows that the partial fraction expansion of $E_{1}(s)$ has the form

$$
E_{1}(s)=E_{1}^{t r}(s)+\frac{a_{1} s+b_{1}}{s^{2}+\alpha^{2}},
$$

where $E_{1}^{t r}(s)$ has poles only in the OLHP and thus contributes only to the transient response. The constants $a_{1}$ and $b_{1}$ are given by

$$
a_{1}=\operatorname{Im}\left(-K_{\alpha}\right), \quad b_{1}=\alpha \operatorname{Re}\left(-K_{\alpha}\right) .
$$

The steady state response $e_{1}^{s s}(t)$ may be evaluated by computing the inverse Laplace transform of the second term on the right-hand side of (15).

(b) If $\arg \left(-K_{\alpha}\right)=0$, then (14) follows from (2).

Our next result uses Theorem 4, together with the fact that all the subsystems in Figure 1 are identical, to show that the steady state tracking errors for each subsystem are identical.

\section{Corollary 1}

(a) Let $e_{k}^{s s}(t)$ denote the steady state tracking error of the $k$ 'th subsystem in Figure 1 in response to the input $r_{1}(t)=t \sin \alpha t$. Then

$$
e_{k}^{s s}(t)=e_{1}^{s s}(t), \quad k=1, \ldots, n .
$$

(b) Suppose in addition that $\arg \left(-K_{\alpha}\right)=0$. Then in steady state, $y_{k}(t)$ is in phase with $r_{1}(t)$ :

$$
y_{k}^{s s}(t)=\left(t-k\left|K_{\alpha}\right|\right) \sin \alpha t, \quad k=1, \ldots, n .
$$


Proof

Theorem 4 shows that $e_{1}^{s s}(t)$ is a sinusoid with frequency $\alpha$, and (a) follows from (5) and the fact that $T^{k-1}(j \alpha)=1$. Together, (2), (14), and (17) yield (b).

Motivated by (18), we say that if $\arg \left(-K_{\alpha}\right)=0$, then the steady state phase error for each oscillator is equal to zero. We now show that if the steady state phase error is nonzero, then the string of oscillators will be string unstable. This is another sufficient condition for string instability.

\section{Theorem 5}

Suppose that $\arg \left(-K_{\alpha}\right) \neq 0$. Then there exists a frequency $\omega$ such that $|T(j \omega)|>1$ and the system of oscillators in Figure 1 is string unstable.

Proof

First consider the case $\arg \left(-K_{\alpha}\right)=\pi$. Then $K_{\alpha}$ is real and positive, and the result follows immediately from (9). Suppose next that $\arg \left(-K_{\alpha}\right) \neq 0, \pi$. Then $K_{\alpha}$ has a nonzero imaginary component. Using the fact that $T(j \alpha)=1$, we have by definition (10) of $K_{\alpha}$ that

$$
K_{\alpha}=\lim _{s \rightarrow j \alpha} \frac{d \log |T(s)|}{d s}+j \lim _{s \rightarrow j \alpha} \frac{d \arg T(s)}{d s} .
$$

Letting $s=\sigma+j \omega$, it follows from the Cauchy-Riemann equations [20, Section 21], [21, p. 41] that

$$
K_{\alpha}=\lim _{\omega \rightarrow \alpha} \frac{\partial \arg T(j \omega)}{\partial \omega}-j \lim _{\omega \rightarrow \alpha} \frac{\partial \log |T(j \omega)|}{\partial \omega} .
$$

Together, the facts that $|T(j \alpha)|=1$ and that $\lim _{\omega \rightarrow \alpha} \frac{\partial \log |T(j \omega)|}{\partial \omega} \neq 0$ imply that there exists a frequency $\omega$ near $\alpha$ such that $|T(j \omega)|>1$.

Theorems 3 and 5 provide two sufficient conditions for string instability. Suppose that neither of these sufficient conditions is satisfied. Then it is easy to find examples of systems that are string stable.

\section{Example 1}

Suppose that $P(s)=1 /\left(s^{2}+\alpha^{2}\right)$ and $C(s)=k s, k>0$. Then $T(s)$ has stable poles, and $K_{\alpha}=-2 / k$, so that $\arg \left(-K_{\alpha}\right)=0$. It is easy to verify that $|T(j \omega)| \leqslant 1, \forall \omega$, and thus the system is string stable.

\subsection{A lower bound on the peak in complementary sensitivity}

Our goal in the present section is to derive a lower bound on $\sup _{\omega}|T(j \omega)|$ that holds whenever the system is assumed to satisfy appropriate performance specifications. If this lower bound exceeds unity, then we may conclude that the system in Figure 1 is string unstable. We will be interested in the case for which neither sufficient condition for string instability derived in Theorems 3 and 5 is satisfied; however, our methods will also yield a lower bound for the case in which $L(s)$ has at least two pairs of poles at $\pm j \alpha$.

We first assume that a specification on the SSE in response to an input $t \sin \alpha t$ must be satisfied.

Assumption 1 (Magnitude Bound on SSE)

Assume that the SSE (13) is uniformly bounded by $q>0$ :

$$
\left|e_{1}^{s s}(t)\right| \leqslant q, \quad \forall t \geqslant 0 .
$$

Recall from Corollary 1 that the SSE for each of the oscillators is identical. The transient error, defined by

$$
e_{i}^{t r}(t) \triangleq e_{i}(t)-e_{i}^{s s}(t)
$$

will in general be different for different oscillators. We assume an IATE performance specification on the sum of the integrals of the absolute values of the transient errors (IATE). 
Assumption 2 (IATE Specification)

Let $e_{i}^{t r}(t)$ in (20) denote the transient error response of the $i$ th oscillator in response to the command $r_{1}(t)=t \sin (\alpha t)$. We assume that the sum of the IATE must satisfy the specification

$$
\sum_{i=1}^{n} \int_{0}^{\infty}\left|e_{i}^{t r}(t)\right| d t \leqslant u(n)
$$

for some positive function $u(n)$.

We now show that Assumptions 1 and 2, combined with one additional hypothesis, imply an upper bound on the gain of $T(j \omega)$.

Lemma 1

Suppose that Assumptions 1 and 2 are satisfied.

(a) Assume in addition that $C(s) P(s)$ possesses one pair of poles at $\pm j \alpha$, and that the phase error is zero: $\arg \left(-K_{\alpha}\right)=0$. Then

$$
|T(j \omega)| \leqslant\left(1+\eta(u(n), q, \alpha, \omega)\left(\omega^{2}-\alpha^{2}\right)^{2}\right)^{\frac{1}{2 n}},
$$

where

$$
\eta(u(n), q, \alpha, \omega)=\frac{u(n)}{\alpha \omega}+\frac{n^{2} q^{2}}{4 \omega^{2}}+\left|\omega^{2}-\alpha^{2}\right| \frac{n u(n) q}{2 \alpha \omega^{2}}+\left(\omega^{2}-\alpha^{2}\right)^{2} \frac{u(n)^{2}}{2 \alpha^{2} \omega^{2}} .
$$

(b) Assume instead that $C(s) P(s)$ possesses at least two pairs of poles at $\pm j \alpha$. Then

$$
|T(j \omega)| \leqslant\left(1+\frac{\left(\omega^{2}-\alpha^{2}\right)^{2}}{2 \alpha \omega} u(n)\right)^{\frac{1}{n}} .
$$

Proof

(a) The assumption that $\arg \left(-K_{\alpha}\right)=0$ implies that $a_{1}=0$ and $b_{1}=-\alpha K_{\alpha}$, where $a_{1}$ and $b_{1}$ are defined in (16). Hence, the Laplace transform of each SSE satisfies

$$
E_{i}^{s s}(s)=\frac{b_{1}}{s^{2}+\alpha^{2}}
$$

Furthermore, it follows from Assumption 1 and (13) that $\left|b_{1}\right| \leqslant \alpha q$. Recalling from Corollary 1 that the SSE is identical for each oscillator, we have, for each $i \geqslant 1$, that

$$
\int_{0}^{\infty} e^{-s t} e_{i}^{t r}(t) d t=T(s)^{i-1}(1-T(s)) \frac{2 \alpha s}{\left(s^{2}+\alpha^{2}\right)^{2}}-\frac{b_{1}}{s^{2}+\alpha^{2}} .
$$

The sum of all $n$ error signals satisfies

$$
\sum_{i=1}^{n} \int_{0}^{\infty} e^{-s t} e_{i}^{t r}(t) d t=\left(1-T(s)^{n}\right) \frac{2 \alpha s}{\left(s^{2}+\alpha^{2}\right)^{2}}-\frac{n b_{1}}{s^{2}+\alpha^{2}} .
$$

Re-arranging the previous equation yields

$$
T(s)^{n}=1-\frac{\left(s^{2}+\alpha^{2}\right)^{2}}{2 \alpha s}\left(\frac{n b_{1}}{s^{2}+\alpha^{2}}+\sum_{i=1}^{n} \int_{0}^{\infty} e^{-s t} e_{i}^{t r}(t) d t\right),
$$

and evaluating this equation at $s=j \omega$ gives

$$
T(j \omega)^{n}=1-\frac{\left(\omega^{2}-\alpha^{2}\right)^{2}}{j 2 \alpha \omega}\left(\frac{-n b_{1}}{\omega^{2}-\alpha^{2}}+\sum_{i=1}^{n} \int_{0}^{\infty} e^{-j \omega t} e_{i}^{t r}(t) d t\right) .
$$


Using Euler's formula, we have

$$
\begin{gathered}
T(j \omega)^{n}=1+\frac{\left(\omega^{2}-\alpha^{2}\right)^{2}}{2 \alpha \omega} \sum_{i=1}^{n} \int_{0}^{\infty} \sin (\omega t) e_{i}^{t r}(t) d t+j\left(\frac{\left(\omega^{2}-\alpha^{2}\right)^{2}}{2 \alpha \omega} \sum_{i=1}^{n} \int_{0}^{\infty} \cos (\omega t) e_{i}^{t r}(t) d t\right. \\
\left.-\frac{n b_{1}\left(\omega^{2}-\alpha^{2}\right)}{2 \alpha \omega}\right) .
\end{gathered}
$$

Furthermore, taking the absolute value, using the fact that $\left|b_{1}\right| \leqslant \alpha q$, and invoking (21) from Assumption 2 yield

$$
\begin{aligned}
|T(j \omega)|^{2 n}= & \left(1+\frac{\left(\omega^{2}-\alpha^{2}\right)^{2}}{2 \alpha \omega} \sum_{i=1}^{n} \int_{0}^{\infty} \sin (\omega t) e_{i}^{t r}(t) d t\right)^{2} \\
& +\left(\frac{\left(\omega^{2}-\alpha^{2}\right)^{2}}{2 \alpha \omega} \sum_{i=1}^{n} \int_{0}^{\infty} \cos (\omega t) e_{i}^{t r}(t) d t-\frac{n b_{1}\left(\omega^{2}-\alpha^{2}\right)}{2 \alpha \omega}\right)^{2} \\
\leqslant & \left(1+\frac{\left(\omega^{2}-\alpha^{2}\right)^{2}}{2 \alpha \omega} u(n)\right)^{2}+\left(\frac{\left(\omega^{2}-\alpha^{2}\right)^{2}}{2 \alpha \omega} u(n)+\frac{n q\left|\omega^{2}-\alpha^{2}\right|}{2 \omega}\right)^{2} \\
= & 1+\left(\omega^{2}-\alpha^{2}\right)^{2}\left(\frac{u(n)}{\alpha \omega}+\frac{n^{2} q^{2}}{4 \omega^{2}}\right)+\left|\omega^{2}-\alpha^{2}\right|^{3} \frac{n u(n) q}{2 \alpha \omega^{2}}+\left(\omega^{2}-\alpha^{2}\right)^{4} \frac{u(n)^{2}}{2 \alpha^{2} \omega^{2}},
\end{aligned}
$$

from which (22) follows immediately.

(b) If the open loop transfer function $C(s) P(s)$ has at least two pairs of complex poles at $\pm j \alpha$, then $E_{i}^{s s}(s)$ defined in (24) is identically zero. Using this fact and following steps similar to those used to prove (22) yields (23).

In either case, $T(j \alpha)=1$ due to the presence of the oscillator poles. The bounds (22) and (23) constrain the rate at which $|T(j \omega)|$ converges to one as $\omega$ approaches $\alpha$, and are a consequence of the requirement (21) that the transient response converges rapidly to zero.

The following assumption implies that the system in Figure 1 has the ability to track low frequency commands with a specified error. On the other hand, although Lemma 1 is applicable for any frequency, system properties in certain frequency ranges tend to be dominated by additional constraints and limitations. For example, at low frequency $T(s)$ may be required to approximate a unity gain low-pass filter. Hence, we make the following assumption for the low frequency behavior of $T(s)$.

Assumption 3 (Low Frequency Behavior)

Let $0<\omega_{l}<\alpha$. For $\omega \in\left(0, \omega_{l}\right)$, the following inequality holds

$$
\left|T(j \omega)^{n}-1\right|<\epsilon,
$$

where $0 \leqslant \epsilon<1$.

Finally, we assume that the system satisfies a bandwidth limitation.

Assumption 4 (Bandwidth Limitation)

The transfer function $T(s)$ obeys the high-frequency roll-off constraint

$$
|T(j \omega)| \leqslant\left(\frac{\omega_{h}}{\omega}\right)^{r}, \quad \text { for all } \omega>\omega_{h}
$$

for some $\omega_{h}>\alpha$ and relative degree $r \geqslant 1$. 
The following theorem shows that Assumptions 1-4, together with one additional hypothesis, imply the existence of a lower bound on the peak magnitude response of the complementary sensitivity function (3).

\section{Theorem 6}

Suppose that Assumptions 1-4 are satisfied.

(a) Assume in addition that $C(s) P(s)$ possesses one pair of poles at $\pm j \alpha$, and that the phase error is zero: $\arg \left(-K_{\alpha}\right)=0$. Then for any $\omega_{m} \in\left(\alpha, \omega_{h}\right)$, we have the following inequality:

$$
\max _{\omega \in\left[\omega_{m}, \omega_{h}\right]} \log |T(j \omega)| \geqslant \frac{\Omega_{H}-\Omega_{\alpha}-\Omega_{L}-\frac{\pi}{2} q+\pi \sum_{i=1}^{N_{z}}\left(\frac{z_{i}}{z_{i}^{2}+\alpha^{2}}\right)+\frac{\pi}{2} \tau}{\int_{\omega_{m}}^{\omega_{h}} W(\omega, \alpha) d \omega},
$$

where $\Omega_{L}, \Omega_{\alpha}$, and $\Omega_{H}$ are bounds on the integral of $\log |T(j \omega)|$ over different frequency ranges:

$$
\begin{gathered}
\Omega_{L} \triangleq \frac{1}{n} \int_{0}^{\omega_{l}} \log (1+\epsilon) W(\omega, \alpha) d \omega \\
\Omega_{\alpha} \triangleq \frac{1}{2 n} \int_{\omega_{l}}^{\omega_{m}} \log \left(1+\eta(u(n), q, \alpha, \omega)\left(\omega^{2}-\alpha^{2}\right)^{2}\right) W(\omega, \alpha) d \omega, \\
\Omega_{H} \triangleq r \int_{\omega_{h}}^{\infty} \log \frac{\omega}{\omega_{h}} W(\omega, \alpha) d \omega .
\end{gathered}
$$

(b) Assume instead that $C(s) P(s)$ possesses at least two pairs of poles at $\pm j \alpha$. Then $T(s)$ must satisfy the lower bound (27), where $\Omega_{H}$ and $\Omega_{L}$ are as defined in (28) and (30), and

$$
\Omega_{\alpha} \triangleq \frac{1}{n} \int_{\omega_{l}}^{\omega_{m}} \log \left(1+\frac{\left(\omega^{2}-\alpha^{2}\right)^{2}}{2 \alpha \omega} u(n)\right) W(\omega, \alpha) d \omega
$$

Proof

We establish this result by splitting the integration interval in (9). In particular,

$$
\begin{aligned}
\int_{\omega_{m}}^{\omega_{h}} \log |T(j \omega)| W(\omega, \alpha) d \omega= & -\int_{0}^{\omega_{l}} \log |T(j \omega)| W(\omega, \alpha) d \omega-\int_{\omega_{l}}^{\omega_{m}} \log |T(j \omega)| W(\omega, \alpha) d \omega \\
& -\int_{\omega_{h}}^{\infty} \log |T(j \omega)| W(\omega, \alpha) d \omega+\frac{\pi}{2} \operatorname{Re}\left(K_{\alpha}\right) \\
& +\pi \sum_{i=1}^{N_{z}}\left(\frac{z_{i}}{z_{i}^{2}+\alpha^{2}}\right)+\frac{\pi}{2} \tau
\end{aligned}
$$

It follows from Assumption 3 and the triangle inequality that

$$
-\int_{0}^{\omega_{l}} \log |T(j \omega)| W(\omega, \alpha) d \omega \geqslant-\Omega_{L} .
$$

Similarly, Lemma 1 implies that

$$
-\int_{\omega_{l}}^{\omega_{m}} \log |T(j \omega)| W(\omega, \alpha) d \omega \geqslant-\Omega_{\alpha},
$$


where $\Omega_{\alpha}$ is defined either by (29) or (31). Together, Assumption 1 and (13) imply that $\operatorname{Re}\left(K_{\alpha}\right) \geqslant$ $-q$. Also note

$$
\int_{\omega_{m}}^{\omega_{h}} \log |T(j \omega)| W(\omega, \alpha) d \omega \leqslant \max _{\omega \in\left[\omega_{m}, \omega_{h}\right]}\{\log |T(j \omega)|\} \int_{\omega_{m}}^{\omega_{h}} W(\omega, \alpha) d \omega .
$$

The result follows by combining the preceding inequalities and applying the high-frequency bound (26).

It follows from Theorem 6 that time and frequency domain specifications, such as those in Assumptions 1-4, impose a lower bound on the peak value of $|T(j \omega)|$. For case (a), should this lower bound prove to be greater than unity, then it provides another sufficient condition for string instability. For case (b), already known to be string unstable, the lower bound provides an estimate of the severity of the instability.

In fact, the lower bound (27) is conservative for the purpose of predicting string instability in case (a). To see this, note that the first two terms on the right-hand side of (32) will be nonnegative if $|T(j \omega)| \leqslant 1$ in the frequency range $\left(0, \omega_{m}\right)$. (If $|T(j \omega)|>1$ in this frequency range, then the system is known to be string unstable without considering behavior at other frequencies.) Hence, we have the following corollary to the proof of Theorem 6. For purposes of simplicity, we also assume that $L(s)$ has no ORHP zeros and the time delay $\tau$ is zero.

Corollary 2

In addition to the hypotheses of Theorem 6 , assume that $|T(j \omega)| \leqslant 1, \forall \omega \in\left(0, \omega_{m}\right)$, and that $N_{z}=0, \tau=0$. Then, for any $\omega_{m} \in\left(\alpha, \omega_{H}\right)$, we have that

$$
\max _{\omega \in\left[\omega_{m}, \omega_{h}\right]} \log |T(j \omega)| \geqslant \frac{\Omega_{H}-\frac{\pi}{2} q}{\int_{\omega_{m}}^{\omega_{h}} W(\omega, \alpha) d \omega} .
$$

It follows immediately from (33) that a necessary condition for string stability is that

$$
q>\frac{2}{\pi} \Omega_{H}
$$

where $\Omega_{H}$ is defined by (30) and $q$ is defined in Assumption 1. If (34) is not satisfied, then the limit as $\omega_{m} \rightarrow \omega_{h}$ of the right-hand side of (33) is equal to infinity, and thus the specifications are infeasible. Hence, the desirability of string stability imposes a tradeoff between bandwidth limitations of the form imposed in Assumption 4, and steady state tracking error requirements as imposed in Assumption 1.

\section{HETEROGENEOUS FEEDBACK LOOP AND EXTENDED COMMUNICATION RANGE}

The string instability analysis in the previous section is limited to the homogeneous oscillator string with predecessor-following control architecture depicted in Figure 1. It cannot be applied to heterogeneous oscillator strings where the controllers can be designed differently. In such a system, there is no complementary sensitivity function $T(s)$ in (4) that describes how the error signal is amplified from one oscillator to its successor. Hence, the heterogeneous oscillator string avoids error amplification at the same frequency, and some of the existing works use this approach to improve string stability [22]. Other methods in the area of vehicle platooning to improve and even regain string stability include speed-dependent separation policy and extended communication ranges. We want to extend our analysis in the previous section to include heterogeneous feedback loop design, a new tracking policy, and an extended communication range. For simplicity, we also assume there is no time delay in the feedback loop. 


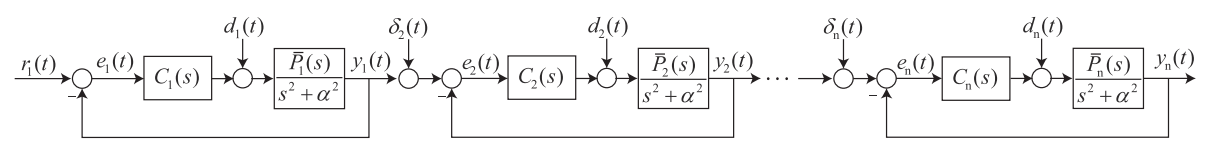

Figure 2. Block diagram depicting an oscillator string with time headway.

\subsection{Time headway operator for harmonic oscillators}

We start our analysis by introducing a new separation policy for a heterogeneous oscillator string shown in Figure 2. Here, the plants can be different, and each contains the dynamics of a harmonic oscillator with natural frequency $\alpha$ radians/second. We write the scalar transfer function $P_{i}(s)$ as

$$
P_{i}(s)=\bar{P}_{i}(s) \frac{1}{s^{2}+\alpha^{2}}, \quad \text { for } i=1,2, \cdots, n,
$$

where $\bar{P}_{i}(s)$ has no zeros at $s= \pm j \alpha$. We also assume the controllers can be designed differently and each controller has harmonic oscillator dynamics. That is

$$
C_{i}(s)=\bar{C}_{i}(s) \frac{1}{s^{2}+\alpha^{2}}, \quad \text { for } i=1,2, \cdots, n .
$$

Because of the presence of the poles at $\pm j \alpha$ in the controller dynamics, the system can achieve asymptotically zero tracking errors for any ramp-enveloped sinusoidal signal of the form $r_{1}(t)=$ $(A t+B) \sin (\alpha t)$ asymptotically and perfect rejection of any disturbance signal $d_{i}(t)$ of the form $A \sin (\alpha t)$ [23]. Theorem 3 has shown that if the oscillator string is homogeneous, the whole system is string unstable if $\delta_{i}(t)$ in Figure 2 is zero. $\delta_{i}(t)$ is the desired distance that the oscillator is kept with its predecessor, and this signal represents the separation policy between oscillators.

We can design $\delta_{i}(t)$ to improve the string stability of the whole system. The concept is similar to the time headway in vehicle platooning, that is to make the intervehicle spacing increase linearly with the vehicle's own velocity. In this section, we limit our discussion to track the ramp-enveloped sinusoidal signal:

$$
r_{1}(t)=\left(\delta_{p}+\delta_{v} t\right) \sin (\alpha t) .
$$

Here, we term $\delta_{v}$ the amplitude velocity of this ramp-enveloped sinusoidal signal. Further, we define the following time headway operator $\nabla_{\mathrm{TH}}$ :

$$
\nabla_{\mathrm{TH}}[f(t)] \triangleq \frac{1}{2} \int_{0}^{t}\left(\frac{d^{2}}{d \tau^{2}} f(\tau)+\alpha^{2} f(\tau)\right) d \tau
$$

where $f(t)$ is assumed twice differentiable. It follows that

$$
\nabla_{\mathrm{TH}}\left[\left(\delta_{p, i}+\delta_{v} t\right) \sin (\alpha t)\right]=\delta_{v} \sin (\alpha t),
$$

which is not difficult to prove. Note that the transfer function of the time headway operator $\nabla_{\mathrm{TH}}$ is $\frac{s^{2}+\alpha^{2}}{2 s}$. Then we propose the following oscillator separation policy that ensures the amplitude of the sinusoidal spacing signal $\delta_{i}(t)$ linearly increases with the amplitude velocity of $y_{i}(t)$ in steady state:

$$
\delta_{i}(t)=\bar{\delta}_{i}-h_{i} \nabla_{\mathrm{TH}}\left[y_{i}(t)\right] .
$$

Here, $h_{i}$ is the time headway constant for each oscillator, and $\bar{\delta}_{i}$ is a vector of sinusoidal signals of the form $A \sin (\alpha t)$. The separation policy (39) ensures that the amplitude velocities of $y_{i}(t)$ are all identical in steady state. We assume the time headway constants are the same: $h_{i}=h$ for each oscillator in this string. 
With time headway, the complementary sensitivity function $T_{h}(s)$ in Figure 2 is now given by

$$
T_{h}(s)=\frac{P_{i}(s) C_{i}(s)}{1+P_{i}(s) C_{i}(s)\left(1+h \frac{s^{2}+\alpha^{2}}{2 s}\right)} .
$$

The transfer function $T_{h}(s)$ describes how errors propagate in a manner similar to $T(s)$ in (5). Applying Theorem 2 to the new $T_{h}(s)$ yields the following integral: ${ }^{*}$

$$
\int_{0}^{\infty} \log \left|T_{h}(j \omega)\right| W(\omega, \alpha) d \omega \geqslant-\frac{\pi}{2} h
$$

The integral in the preceding text shows that string stability is potentially feasible because the righthand side is negative due to the time headway operator.

\subsection{Multivariable representation of the oscillator string}

We also want to consider the more general communication range adopted in [11] and assume that the oscillators are permitted to communicate with a few neighbors forward and backward. This is different from the communication range used in Figures 1 and 2. With the extended communication strategies, the transfer function from a disturbance at the lead oscillator to the error in the $n$th oscillator is no longer equal to the product of the individual transfer functions. Hence, in this section, we examine the disturbance propagation from the first oscillator to the last one for an arbitrarily large string of oscillators. Doing so requires us to use a multivariable representation of the oscillator string.

We augment the output, control, error, separation, and disturbance variables as

$$
\begin{aligned}
& \underline{y}(t)=\left[\begin{array}{llll}
y_{1}(t) & y_{2}(t) & \cdots & y_{n}(t)
\end{array}\right]^{\mathrm{T}}, \\
& \underline{u}(t)=\left[\begin{array}{llll}
u_{1}(t) & u_{2}(t) & \cdots & u_{n}(t)
\end{array}\right]^{\mathrm{T}}, \\
& \underline{e}(t)=\left[\begin{array}{llll}
e_{1}(t) & e_{2}(t) & \cdots & e_{n}(t)
\end{array}\right]^{\mathrm{T}}, \\
& \underline{d}(t)=\left[\begin{array}{llll}
d_{1}(t) & d_{2}(t) & \cdots & d_{n}(t)
\end{array}\right]^{\mathrm{T}}, \\
& \underline{\delta}(t)=\left[\begin{array}{llll}
0 & \delta_{2}(t) & \cdots & \delta_{n}(t)
\end{array}\right]^{\mathrm{T}} .
\end{aligned}
$$

We make the following assumptions by considering the extended communication ranges, heterogeneous feedback loop, and the new separation policy.

\section{Assumption 5 (Communication Range)}

We assume that the $i$ th oscillator is permitted to communicate with $c_{f}$ oscillators in front and $c_{r}$ oscillators behind itself. Here $c_{r}, c_{f}$ are fixed natural numbers and $c_{f} \geqslant 1$. Further, for simplicity, we assume the number of oscillators $n$ to be divisible by the forward communication range $c_{f}$, which is $n=N c_{f}$.

\section{Assumption 6 (Heterogeneous Feedback Loop)}

Considering the extended communication range in Assumption 5, the control policy can be written using a multivariable transfer function matrix $\mathcal{C}(s)$, where $\mathcal{C}(s)$ is a $\left(c_{f}, c_{r}\right)$-banded transfer matrix. That is

$$
\underline{u}(t)=\mathcal{C}(s) \star \underline{e}(t)
$$

\footnotetext{
¥It follows from the proof of Theorem 2 that the integral relation (9) holds for any stable, proper, rational transfer function $T(s)$ that satisfies $T(j \alpha)=1$. Hence (a) holds for $T_{h}(s)$ defined in (40).
} 
where $\underline{u}(t)$ and $\underline{e}(t)$ are the augmented control and error signals defined in (41). In addition, we assume each nonzero element in $\mathcal{C}(s)$ contains the dynamics of a harmonic oscillator with natural frequency $\alpha$ radians/second. Thus, we write $\mathcal{C}(s)$ as

$$
\mathcal{C}(s)=\overline{\mathcal{C}}(s) \frac{1}{s^{2}+\alpha^{2}},
$$

with $\overline{\mathcal{C}}(j \alpha)$ non-singular.

Assumption 7 (Oscillator Separation Policy)

We adopt the separation policy in (39). Further, we define $H$, the matrix of time headway, as $H=\operatorname{diag}\left\{h_{i}\right\} \geqslant 0$, and $\underline{\delta}_{0}=\left[\begin{array}{ll}0 & \bar{\delta}_{2} \cdots \bar{\delta}_{n}\end{array}\right]^{\mathrm{T}}$ to be a vector of sinusoidal signals of the form $A \sin (\alpha t)$. The separation policy can be rewritten as

$$
\underline{\delta}(t)=\underline{\delta}_{0}-H \nabla_{\mathrm{TH}}[\underline{y}(t)],
$$

where $\underline{y}(t)$ and $\underline{\delta}(t)$ are the augmented output and separation signals defined in (41).

To describe the oscillator string under Assumptions 5-7, we first define the multivariable plant transfer function: $\mathcal{P}(s)=\operatorname{diag}\left\{P_{i}(s)\right\}, \overline{\mathcal{P}}(s)=\operatorname{diag}\left\{\bar{P}_{i}(s)\right\}$. Then equation (35) can be rewritten as

$$
\underline{y}(t)=\mathcal{P}(s) \star \underline{u}(t),
$$

where $\mathcal{P}(s)$ can be factored as

$$
\mathcal{P}(s)=\overline{\mathcal{P}}(s) \frac{1}{s^{2}+\alpha^{2}} .
$$

Similarly, we define the error signal as

$$
\underline{e}(t)=\underline{\delta}(t)-M \underline{y}(t)+V_{1}^{n} r_{1}(t),
$$

where $V_{1}^{n}=\left[\begin{array}{llll}1 & 0 & \cdots & 0\end{array}\right]^{\mathrm{T}}$ and $M$ denotes the coupling matrix

$$
M=\left[\begin{array}{cccc}
1 & 0 & \cdots & 0 \\
-1 & 1 & \cdots & 0 \\
\vdots & \ddots & \ddots & 0 \\
0 & \cdots & -1 & 1
\end{array}\right]
$$

The multivariable system representation of the oscillator string is shown in Figure 3. Using (42)(45), the output variable $y(t)$ can be related to the target separation variable $\underline{\delta}_{0}$ and the lead oscillator target position $r_{1}(t)$ by the following equation

$$
\underline{y}(t)=(I+\mathcal{L}(s))^{-1} \mathcal{P}(s) \mathcal{C}(s) \star\left(\underline{\delta}_{0}+V_{1}^{n} r_{1}(t)\right),
$$

where

$$
\mathcal{L}(s)=\mathcal{P}(s) \mathcal{C}(s)\left(M+\frac{s^{2}+\alpha^{2}}{2 s} H\right)
$$

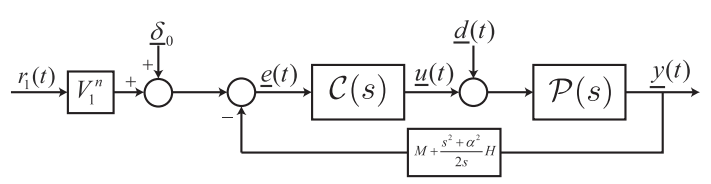

Figure 3. Multivariable feedback loop representation of the oscillator string. 
Hence, we can define the closed-loop multivariable transfer function matrix $\mathcal{H}_{y r}(s)$ as

$$
\mathcal{H}_{y r}(s)=(I+\mathcal{L}(s))^{-1} \mathcal{P}(s) \mathcal{C}(s)=\left(I-(I+\mathcal{L}(s))^{-1}\right)\left(M+\frac{s^{2}+\alpha^{2}}{2 s} H\right)^{-1} .
$$

Assumptions 5-7 allow us to establish some properties of the closed-loop matrix $\mathcal{H}_{y r}(s)$ at frequency $\alpha$.

Lemma $2\left(\right.$ Values of $\mathcal{H}_{y r}$ at $\left.s=j \alpha\right)$

Consider $\mathcal{H}_{y r}$ as defined in (49). Then subject to Assumptions 5-7, we have

$$
\begin{gathered}
\mathcal{H}_{y r}(j \alpha)=M^{-1}, \\
\mathcal{H}_{y r}^{\prime}(j \alpha)=-M^{-1} H M^{-1} .
\end{gathered}
$$

Proof

From the definition of $\mathcal{H}_{y r}(s)$ in (49), we have

$$
\begin{aligned}
\mathcal{H}_{y r}(s) & =\left[I+\mathcal{P}(s) \mathcal{C}(s)\left(M+\frac{s^{2}+\alpha^{2}}{2 s} H\right)\right]^{-1} \mathcal{P}(s) \mathcal{C}(s) \\
& =\left(M^{-1}(\mathcal{P}(s) \mathcal{C}(s))^{-1}+I+\frac{s^{2}+\alpha^{2}}{2 s} M^{-1} H\right)^{-1} M^{-1} \\
& =\left[I+\left(s^{2}+\alpha^{2}\right)^{2} M^{-1}(\overline{\mathcal{P}}(s) \overline{\mathcal{C}}(s))^{-1}+\frac{s^{2}+\alpha^{2}}{2 s} M^{-1} H\right]^{-1} M^{-1} .
\end{aligned}
$$

Evaluating (52) at $s=j \alpha$ gives (50) and differentiating (52) at $s=j \alpha$ gives (51).

The lower left element of $\mathcal{H}_{y r}(s)$ describes the response of the output of the last oscillator to an output disturbance at the first oscillator:

$$
\mathcal{H}_{y_{n} r_{1}}(s)=\left(V_{n}^{n}\right)^{\mathrm{T}} \mathcal{H}_{y r}(s) V_{1}^{n},
$$

where $V_{1}^{n}$ and $V_{n}^{n}$ are the 1 st and $n$th canonical basis vectors, respectively. We then apply Theorem 2 to the transfer function $\mathcal{H}_{y_{n} r_{1}}(s)$ and obtain the following theorem.

\section{Theorem 7}

Consider $\mathcal{H}_{y_{n} r_{1}}(s)$ as defined in (53). Then subject to Assumptions 5-7, we have

$$
\int_{0}^{\infty} \log \left|\mathcal{H}_{y_{n} r_{1}}(j \omega)\right| W(\omega, \alpha) d \omega \geqslant-\frac{\pi}{2} n \bar{h},
$$

where $\bar{h}$ is the average time headway

$$
\bar{h}=\frac{1}{n} \sum_{i=1}^{n} h_{i} .
$$

Proof

Note from the definition of $M$ in (46) that

$$
M^{-1}=\left[\begin{array}{cccc}
1 & 0 & \cdots & 0 \\
1 & 1 & \cdots & 0 \\
\vdots & \ddots & \ddots & 0 \\
1 & \cdots & 1 & 1
\end{array}\right] .
$$

Then from Lemma 2, we have

$$
\mathcal{H}_{y_{n} r_{1}}(j \alpha)=1 \text {, and } \mathcal{H}_{y_{n} r_{1}}^{\prime}(j \alpha)=-n \bar{h} \text {. }
$$

The result then follows by using (9). 


\subsection{Lower bound on disturbance amplification}

In this section, we present a lower bound on the worst case disturbance amplification along the string when the system is assumed to satisfy certain communication constraints, a high-frequency bandwidth limitation, and certain transient performance in response to a ramp-enveloped sinusoidal signal. If this lower bound grows at least linearly with the number of oscillators $n$, then we may conclude that the system in Figure 3 is string unstable.

We first present some assumptions on the system structural properties induced by the communications range and high-frequency bandwidth limitation. From Assumption 5, it is easy to show $\mathcal{L}(s)$ is a $\left(c_{f}, c_{r}\right)$-banded transfer matrix. Then $\mathcal{L}(s)$ can be written as an $N \times N$ block matrix, with $N=n / c_{f}$ :

$$
\mathcal{L}(s)=\left[\begin{array}{ccccc}
\mathcal{L}_{1,1}(s) & \mathcal{L}_{1,2}(s) & \mathcal{L}_{1,3}(s) & \ldots & 0 \\
\mathcal{L}_{2,1}(s) & \mathcal{L}_{2,2}(s) & \mathcal{L}_{2,3}(s) & \ldots & 0 \\
0 & \mathcal{L}_{3,2}(s) & \mathcal{L}_{3,3}(s) & \ddots & \vdots \\
\vdots & \ddots & \ddots & \ddots & \mathcal{L}_{N-1, N}(s) \\
0 & \cdots & 0 & \mathcal{L}_{N, N-1}(s) & \mathcal{L}_{N, N}(s)
\end{array}\right]
$$

where each block element $\mathcal{L}_{i, j}(s)$ is a $c_{f} \times c_{f}$ dimensional transfer function matrix, and $\mathcal{L}_{i, j}(s)=0$ for $j>i+l_{r}$, where $l_{r}=\left\lceil c_{r} / c_{f}\right\rceil$ is the communication range ratio introduced in Assumption 5 .

It follows that $I+\mathcal{L}(s)$ can be conveniently factorized in a block Lower Upper (LU) form described in the following lemma [11].

Lemma 3 (Block LU Factorization of $\mathcal{L}(s)$ )

Under Assumption 5, let $\mathcal{L}(s)$ be the $\left(c_{f}, c_{r}\right)$-banded transfer function matrix defined in (56). Then

$$
I+\mathcal{L}(s) \triangleq \mathcal{M}_{L}(s) \mathcal{M}_{U}(s)
$$

where $\mathcal{M}_{U}(s)$ is given as

$$
\mathcal{M}_{U}(s)=\left[\begin{array}{ccccc}
I & \mathcal{U}_{1,2}(s) & \mathcal{U}_{1,3}(s) & \cdots & 0 \\
0 & I & \mathcal{U}_{2,3}(s) & \cdots & 0 \\
0 & 0 & I & \ddots & \vdots \\
\vdots & \ddots & \ddots & \ddots & \vdots \\
0 & \cdots & 0 & 0 & I
\end{array}\right]
$$

and $\mathcal{M}_{L}(s)$ as

$$
\mathcal{M}_{L}(s)=\left[\begin{array}{ccccc}
\tilde{\mathcal{S}}_{1,1}^{-1}(s) & 0 & 0 & \ldots & 0 \\
\mathcal{L}_{2,1}(s) & \tilde{\mathcal{S}}_{2,2}^{-1}(s) & 0 & \ldots & 0 \\
0 & \mathcal{L}_{3,2}(s) & \tilde{\mathcal{S}}_{3,3}^{-1}(s) & \ddots & \vdots \\
\vdots & \ddots & \ddots & \ddots & 0 \\
0 & \ldots & 0 & \mathcal{L}_{N, N-1}(s) \tilde{\mathcal{S}}_{N, N}^{-1}(s)
\end{array}\right]
$$

and $\tilde{\mathcal{S}}_{k, k}$ and $\mathcal{U}_{k, j}$ are defined recursively by

$$
\begin{aligned}
\tilde{S}_{1,1}(s) & =\left(1+\mathcal{L}_{1,1}(s)\right)^{-1} \\
\mathcal{U}_{1, j}(s) & =\tilde{\mathcal{S}}_{1,1}(s) \mathcal{L}_{1, j}(s): j=2,3, \ldots, N \\
\tilde{\mathcal{S}}_{k, k}(s) & =\left(1+\mathcal{L}_{k, k}(s)-\mathcal{L}_{k, k-1}(s) \mathcal{U}_{k-1, k}(s)\right)^{-1}: k=2,3, \ldots, N \\
\mathcal{U}_{k, j}(s) & =\tilde{\mathcal{S}}_{k, k}(s)\left(\mathcal{L}_{k, j}(s)-\mathcal{L}_{k, k-1}(s) \mathcal{U}_{k-1, j}(s)\right): 1<k<j \leqslant N .
\end{aligned}
$$


From these equations, it follows that the multivariable sensitivity function $\mathcal{S}(s)=(I+\mathcal{L}(s))^{-1}$ can be written as a product of upper and lower block triangular matrices

$$
\mathcal{S}(s)=\mathcal{M}_{U}^{-1}(s) \mathcal{M}_{L}^{-1}(s)=\left[\begin{array}{cccc}
I & * & \cdots & * \\
0 & I & \ddots & \vdots \\
\vdots & \ddots & \ddots & * \\
0 & \cdots & 0 & I
\end{array}\right]\left[\begin{array}{cccc}
\tilde{\mathcal{S}}_{1,1}(s) & 0 & \cdots & 0 \\
\tilde{\mathcal{S}}_{2,1}(s) & \tilde{\mathcal{S}}_{2,2}(s) & \ddots & \vdots \\
\vdots & \ddots & \ddots & 0 \\
\tilde{\mathcal{S}}_{N, 1}(s) & \cdots & \tilde{\mathcal{S}}_{N, N-1}(s) & \tilde{\mathcal{S}}_{N, N}(s)
\end{array}\right],
$$

where $*$ denotes possibly nonzero transfer function blocks within the matrix $\mathcal{M}_{U}^{-1}(s)$. Further, we have

$$
\mathcal{S}_{N, i}(s)=\tilde{\mathcal{S}}_{N, i}(s)=\tilde{\mathcal{S}}_{N, N}(s) \prod_{k=i}^{N-1} \tilde{\mathcal{T}}_{k+1, k}(s)
$$

where

$$
\tilde{\mathcal{T}}_{k+1, k}(s) \triangleq-\mathcal{L}_{k+1, k}(s) \tilde{\mathcal{S}}_{k, k}(s) \text { for } k=1,2, \cdots, N-1 .
$$

Further, noting that $\tilde{\mathcal{S}}_{k, k}(s)$ is exactly the lower right-hand block of the multivariable sensitivity function $\mathcal{S}(s)$ in (61), we assume the following uniform bounds on $\tilde{\mathcal{S}}_{k, k}(s)$.

Assumption 8 (Uniform Bounds on $\tilde{\mathcal{S}}_{k, k}(s)$ )

There exists a finite number $\sigma \geqslant 0$ such that

$$
\left\|\tilde{\mathcal{S}}_{k, k}(s)\right\|_{\mathcal{H}_{\infty}} \leqslant \sigma \quad \text { for } k=1,2, \cdots, N .
$$

We also assume each $\tilde{\mathcal{T}}_{k+1, k}(j \omega)$ satisfies a high-frequency bandwidth limitation based on its definition in (63).

\section{Assumption 9 (Loop High-Frequency Bound)}

The loop transfer functions $\tilde{\mathcal{T}}_{k+1, k}(s)$ with $k \in\{1,2, \ldots, N-1\}$, defined in (63), obey the uniform high-frequency bound

$$
\left\|\tilde{\mathcal{T}}_{k+1, k}(j \omega)\right\| \leqslant\left(\frac{\omega_{H}}{\omega}\right)^{r}, \quad \text { for all } \omega>\omega_{H},
$$

for some $\omega_{H}>0$ independent of $N$ and (relative degree) $r \geqslant 1$ and all $k \in\{1,2, \ldots, N-1\}$. In addition, we assume that $\forall \omega \geqslant \omega_{H}$

$$
\left\|(\mathcal{P}(s) \mathcal{C}(s))_{1,1}(j \omega) V_{1}^{c_{f}}\right\| \leqslant \bar{p},
$$

for some $\bar{p}<\infty$.

We now show that Assumptions 8 and 9 imply a bound on the integral of the magnitude response of $\mathcal{H}_{y_{n} r_{1}}(s)$ over a high-frequency range.

Corollary 3

Under Assumptions 5-9, we have

$$
\int_{\omega_{H}}^{\infty} \log \left|\mathcal{H}_{y_{n} r_{1}}(j \omega)\right| W(\omega, \alpha) d \omega \leqslant \log (\sigma \bar{p}) \frac{\omega_{H}}{\omega_{H}^{2}-\alpha^{2}}+\frac{(N-1) r}{2 \alpha} \log \frac{\omega_{H}-\alpha}{\omega_{H}+\alpha} .
$$

\section{Proof}

The proof is similar to the proof of Corollary 5 in [11]. By following the same line of logic, we have the following inequality: 


$$
\left|\mathcal{H}_{y_{n} r_{1}}(j \omega)\right| \leqslant \sigma \bar{p}\left(\frac{\omega_{H}}{\omega}\right)^{r(N-1)}, \quad \text { for all } \omega>\omega_{H}
$$

Then, we have

$$
\begin{aligned}
\int_{\omega_{H}}^{\infty} \log \left|\mathcal{H}_{y_{n} r_{1}}(j \omega)\right| W(\omega, \alpha) d \omega \leqslant & {\left[\log (\sigma \bar{p})+(N-1) r \log \omega_{H}\right] \int_{\omega_{H}}^{\infty} W(\omega, \alpha) d \omega } \\
& -(N-1) r \int_{\omega_{H}}^{\infty} W(\omega, \alpha) \log \omega d \omega .
\end{aligned}
$$

The result in (66) follows by solving the integrals in the inequality in the preceding text.

As $\mathcal{P}(s) \mathcal{C}(s)$ contains a double oscillator dynamics in Assumption 6, the oscillator string can achieve asymptotically zero tracking error for a sinusoidal signal in the form of (37). We assume the oscillator string satisfies a performance specification on the sum of integral absolute errors (IAE) that describes how fast the errors converge to zero.

Assumption 10 (IAE Specification on Transient Response)

For $i=1,2, \cdots, n$, let $e_{i}^{r a m p}(t)$ be the error response of the $i$ th oscillator to a ramp-enveloped sinusoidal signal: $r_{1}(t)=t \sin (\alpha t)$. We assume that for all $n$ oscillators, the integral of the absolute value of $e_{i}^{r a m p}(t)$ is bounded as

$$
\sum_{i=1}^{n} \int_{0}^{\infty}\left|e_{i}^{r a m p}(t)\right| d t \leqslant u(n),
$$

for some positive function $u(n)$.

One immediate consequence of Assumption 10 is a bound on the frequency response of $\mathcal{H}_{y_{n} r_{1}}(s)$.

Lemma 4

Let Assumption 10 hold. Then, for all $\omega \in \mathbb{R}$

$$
\left|\mathcal{H}_{y_{n} r_{1}}(j \omega)\right| \leqslant 1+u(n) \frac{\left(\omega^{2}-\alpha^{2}\right)^{2}}{2 \alpha \omega} .
$$

Proof

This lemma can be proved by following a line similar to the proof of Lemma 6 in [11].

In addition, $\mathcal{H}_{y_{n} r_{1}}(s)$ typically has low-pass characteristics. Hence, we make the following assumption for the low frequency behavior of $\mathcal{H}_{y_{n} r_{1}}(s)$.

Assumption 11 (Low Frequency Behavior)

Let $0<\omega_{L}<\alpha$. For $\omega \in\left(0, \omega_{L}\right)$, the following inequality holds

$$
\left|\mathcal{H}_{y_{n} r_{1}}(j \omega)-1\right|<\epsilon,
$$

where $0 \leqslant \epsilon<1$.

The following theorem provides a lower bound on the peak magnitude response of $\mathcal{H}_{y_{n} r_{1}}(s)$.

Theorem 8

Consider a system subject to Assumptions 5-11. Then for any $\omega_{M} \in\left(\alpha, \omega_{H}\right)$, we have the following inequality:

$$
\max _{\omega \in\left[\omega_{M}, \omega_{H}\right]} \log \left|\mathcal{H}_{y_{n} r_{1}}(j \omega)\right| \geqslant \frac{\bar{\Omega}_{H}-\bar{\Omega}_{\alpha}-\bar{\Omega}_{L}-\frac{\pi}{2} n \bar{h}}{\int_{\omega_{M}}^{\omega_{H}} W(\omega, \alpha) d \omega},
$$


where $\bar{\Omega}_{L}, \bar{\Omega}_{\alpha}, \bar{\Omega}_{H}$ are bounds on different frequency ranges of the integral of $\log \left|\mathcal{H}_{y_{n} r_{1}}(j \omega)\right|$ and defined as

$$
\begin{aligned}
& \bar{\Omega}_{L} \triangleq \int_{0}^{\omega_{L}} \log (1+\epsilon) W(\omega, \alpha) d \omega=-\log (1+\epsilon) \frac{\omega_{L}}{\omega_{L}^{2}-\alpha^{2}} \\
& \bar{\Omega}_{\alpha} \triangleq \int_{\omega_{L}}^{\omega_{M}} \log \left[1+u(n) \frac{\left(\omega^{2}-\alpha^{2}\right)^{2}}{2 \alpha \omega}\right] W(\omega, \alpha) d \omega \\
& \bar{\Omega}_{H} \triangleq-\log (\sigma \bar{p}) \frac{\omega_{H}}{\omega_{H}^{2}-\alpha^{2}}+\frac{(N-1) r}{2 \alpha} \log \frac{\omega_{H}+\alpha}{\omega_{H}-\alpha} .
\end{aligned}
$$

In addition, assume that $\left|\mathcal{H}_{y_{n} r_{1}}(j \omega)\right| \leqslant 1, \forall \omega \in(0, \alpha)$. Then if $\bar{\Omega}_{H}-\frac{\pi}{2} n \bar{h}>0$, we have

$$
\max _{\omega \in\left(\omega_{M}, \omega_{H}\right)}\left|\mathcal{H}_{y_{n} r_{1}}(j \omega)\right| \geqslant \exp \left(\frac{\alpha^{3}\left(\bar{\Omega}_{H}-\frac{\pi}{2} n \bar{h}\right)^{2}}{\omega_{H} u(n)\left(\omega_{H}^{2}+\alpha^{2}\right)}\right) .
$$

Furthermore, if $u(n)<n \bar{u}$ and $n$ is sufficiently large, then

$$
\max _{\omega \in\left(\omega_{M}, \omega_{H}\right)}\left|\mathcal{H}_{y_{n} r_{1}}(j \omega)\right| \geqslant \exp \left(n \beta\left(\alpha, \omega_{H}, \bar{u}, r, c_{f}, \bar{h}\right)\right),
$$

where $\beta\left(\alpha, \omega_{H}, \bar{u}, r, c_{f}, \bar{h}\right)$ represents the lower bound of the growth per oscillator in the peak of the frequency response and is defined as

$$
\beta\left(\alpha, \omega_{H}, \bar{u}, r, c_{f}, \bar{h}\right)=\frac{\alpha^{3}}{\omega_{H} \bar{u}\left(\omega_{H}^{2}+\alpha^{2}\right)}\left(\frac{r}{2 \alpha c_{f}} \log \frac{\omega_{H}+\alpha}{\omega_{H}-\alpha}-\frac{\pi}{2} \bar{h}\right)^{2} .
$$

Proof

The inequality (71) can be proved following the same line of logic as the proof of Theorem 6.

From Lemma 4, we have

$$
\begin{aligned}
\int_{\alpha}^{\omega_{M}} \log \left|\mathcal{H}_{y_{n} r_{1}}(j \omega)\right| W(\omega, \alpha) d \omega & \leqslant \int_{\alpha}^{\omega_{M}} \log \left(1+u(n) \frac{\left(\omega^{2}-\alpha^{2}\right)^{2}}{2 \alpha \omega}\right) \times \frac{\omega^{2}+\alpha^{2}}{\left(\omega^{2}-\alpha^{2}\right)^{2}} d \omega \\
& \leqslant\left(\omega_{M}-\alpha\right)\left(\omega_{H}^{2}+\alpha^{2}\right) \max _{\omega \in\left(\alpha, \omega_{M}\right)} \frac{\log \left(1+u(n) \frac{\left(\omega^{2}-\alpha^{2}\right)^{2}}{2 \alpha \omega}\right)}{\left(\omega^{2}-\alpha^{2}\right)^{2}} .
\end{aligned}
$$

Then it is not difficult to prove

$$
\max _{\omega \in\left(\alpha, \omega_{M}\right)} \frac{\log \left(1+u(n) \frac{\left(\omega^{2}-\alpha^{2}\right)^{2}}{2 \alpha \omega}\right)}{\left(\omega^{2}-\alpha^{2}\right)^{2}}=\frac{u(n)}{2 \alpha^{2}} .
$$

Hence, we obtain

$$
\int_{\alpha}^{\omega_{M}} \log \left|\mathcal{H}_{y_{n} r_{1}}(j \omega)\right| W(\omega, \alpha) d \omega \leqslant \frac{u(n)\left(\omega_{H}^{2}+\alpha^{2}\right)}{2 \alpha^{2}}\left(\omega_{M}-\alpha\right)
$$

Applying (74) to inequality (71) yields the following inequality 


$$
\begin{aligned}
\max _{\omega \in\left(\omega_{M}, \omega_{H}\right)} \log \left|\mathcal{H}_{y_{n} r_{1}}(j \omega)\right| & \geqslant \frac{\bar{\Omega}_{H}-\frac{\pi}{2} n \bar{h}-\frac{u(n)\left(\omega_{H}^{2}+\alpha^{2}\right)}{2 \alpha^{2}}\left(\omega_{M}-\alpha\right)}{\int_{\omega_{M}}^{\infty} W(\omega, \alpha) d \omega} \\
& \geqslant \frac{2 \alpha}{\omega_{H}}\left(\omega_{M}-\alpha\right)\left(\bar{\Omega}_{H}-\frac{\pi}{2} n \bar{h}-\frac{u(n)\left(\omega_{H}^{2}+\alpha^{2}\right)}{2 \alpha^{2}}\left(\omega_{M}-\alpha\right)\right) \\
& =\frac{2 \alpha}{\omega_{H}}\left[-\frac{u(n)\left(\omega_{H}^{2}+\alpha^{2}\right)}{2 \alpha^{2}}\left(\omega_{M}-\alpha\right)^{2}+\left(\bar{\Omega}_{H}-\frac{\pi}{2} n \bar{h}\right)\left(\omega_{M}-\alpha\right)\right]
\end{aligned}
$$

If we take $\omega_{M}=\alpha+\frac{\alpha^{2}\left(\bar{\Omega}_{H}-\frac{\pi}{2} n \bar{h}\right)}{u(n)\left(\omega_{H}^{2}+\alpha^{2}\right)}$, the inequality (72) is obtained.

Theorem 8 shows that under certain conditions, the lower bound of $\max _{\omega \in\left(\omega_{M}, \omega_{H}\right)} \log \left|\mathcal{H}_{y_{n} r_{1}}(j \omega)\right|$ will increase at least linearly with the number of oscillators. Hence, the peak will grow without bound, and the oscillator string will be string unstable.

\section{NUMERICAL EXAMPLES}

We present a few examples to illustrate the results.

\subsection{Homogeneous oscillator string}

We present a numerical example to illustrate the results in Theorem 6. Consider a string of $n$ identical oscillators with frequency $\alpha=1$ and plant transfer function $P(s)=(s+0.5) /\left(s^{2}+1\right)$. A controller that achieves zero steady state phase error, $\arg \left(-K_{\alpha}\right)=0$, is given by

$$
C(s)=\frac{40(s+10)(s+2)}{s^{2}+0.05 s+1.5} .
$$

A plot of the lower bound (27) as a function of $\omega_{h}$, the frequency at which the bandwidth limitation becomes effective, is given in Figure 4 for various values of the parameter $q$ that governs the size of the tracking error via (19). As expected, smaller values of $\omega_{h}$ increase the size of the lower bound, and for a given value of $\omega_{h}$, the bound increases as the constraint on the tracking error decreases.

The corresponding complementary sensitivity function is

$$
T(s)=\frac{40 s^{3}+500 s^{2}+1040 s+400}{s^{4}+40.05 s^{3}+502.5 s^{2}+1040 s+401.5} .
$$

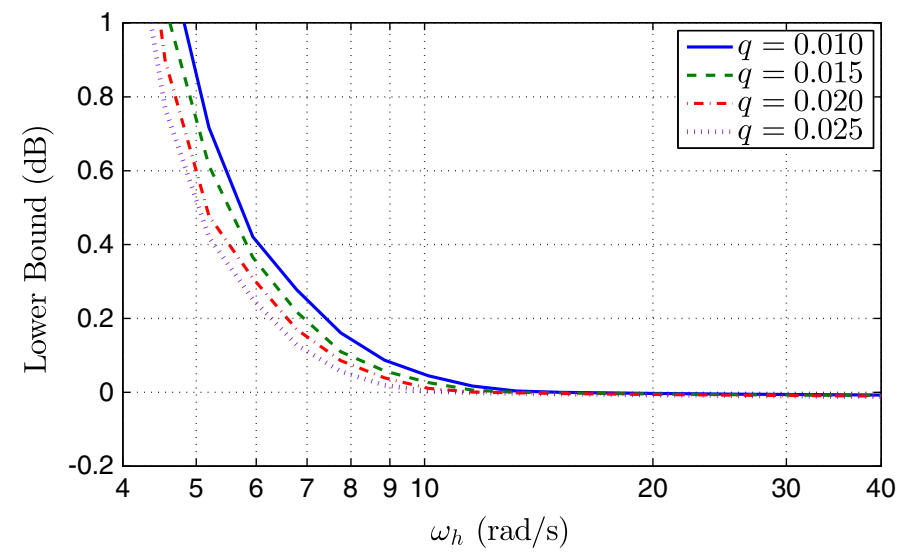

Figure 4. The lower bound (27) versus $\omega_{h}$, for parameters $n=10, r=1, \epsilon=0.1, u(10)=1$, and $\omega_{l}=0.6$. 


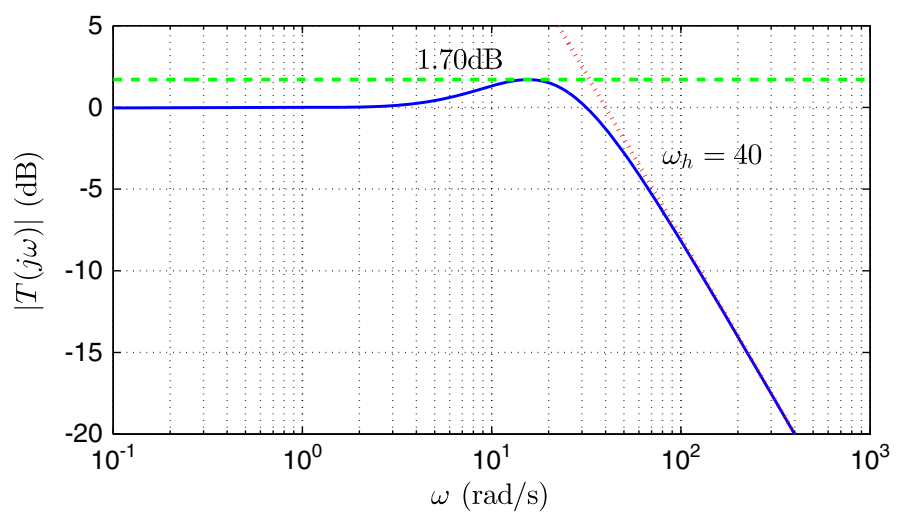

Figure 5. Magnitude response of $T(s)$.

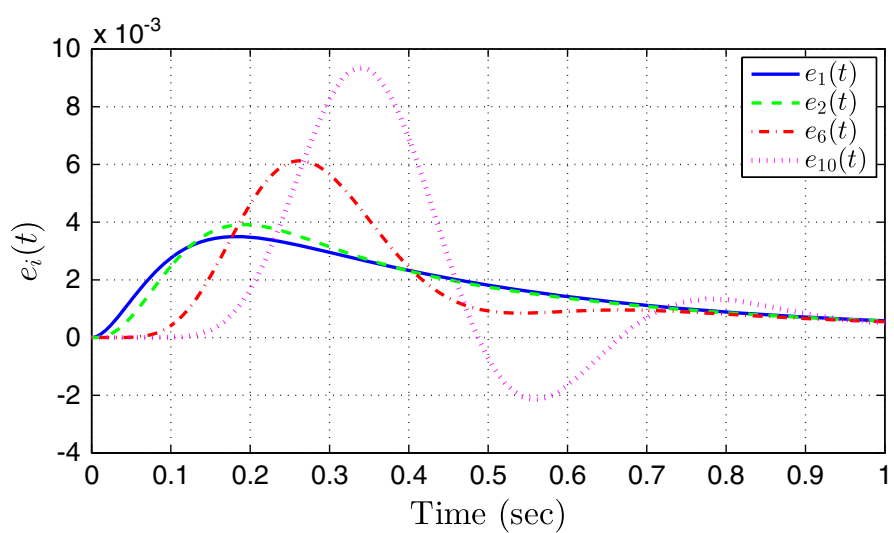

Figure 6. Tracking errors $e_{i}(t)$ defined in (2).

Table I. Parameters to calculate the lower bound.

\begin{tabular}{cccccccc}
\hline$n$ & $\epsilon$ & $u(n)$ & $q$ & $r$ & $\omega_{h}$ & $\omega_{l}$ & $\omega_{m}$ \\
\hline 10 & 0.0367 & 0.072 & 0.001 & 1 & 40 & 0.536 & 1.95 \\
\hline
\end{tabular}

As it happens, the DC gain of $|T(j \omega)|$ is nearly unity, and it is straightforward to verify that $T(j 1)=1$ and $K_{\alpha}=-0.001$. The Bode magnitude plot for (76), depicted in Figure 5, shows a peak value of $1.70 \mathrm{~dB}$, or 1.22 in absolute terms. As a consequence, the string of oscillators is string unstable. The tracking errors (2) in response to an input $r_{1}(t)=t \sin t$ are plotted in Figure 6, and show transient peaks that, as expected, increase in magnitude along the string. In all cases, the steady state value of the tracking error is given by $e_{k}^{s S}(t)=0.001 \sin t$, as predicted from Theorem 4 and Corollary 1.

To illustrate the bound (27), we find that the various parameters used to construct the bound have the values depicted in Table I. With these parameter values, we predict that $|T(j \omega)|$ must have a peak greater than $1.0146(0.126 \mathrm{~dB})$, which is less than the observed peak value of $1.70 \mathrm{~dB}$. The difference is due in part to conservativeness in the lower bound (27), and in part due to controller design. A different controller might yield a smaller peak, but no smaller than the guaranteed lower bound provided that the rest of the design satisfies the parameter values from Table I.

\subsection{Heterogeneous oscillator string}

We will illustrate the result in Theorem 8 in this section. Instead of providing a design example, we show the lower bound on the peak growth of the frequency response per oscillator for different 


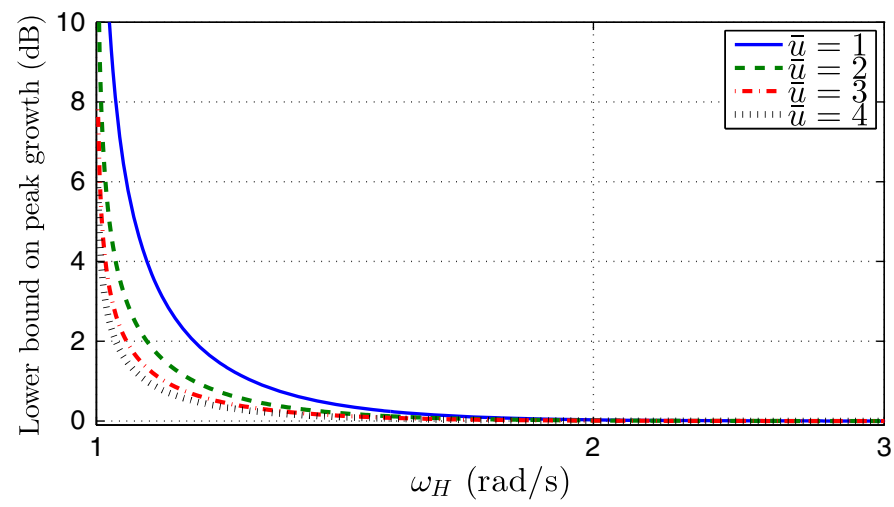

Figure 7. Lower bound on peak growth of magnitude response per oscillator versus $\omega_{H}$ for different values of $\bar{u}$.

bandwidth limitations $\omega_{H}$ and IAE specifications $\bar{u}$ in (73). The plot is shown in Figure 7. The parameter values are $r=1, c_{f}=2, \alpha=1$, and $\bar{h}=0.1$.

We can see from Figure 7 that the lower bound on peak growth per oscillator in (73) $(20 \times$ $\beta\left(\alpha, \omega_{H}, \bar{u}, r, c_{f}, \bar{h}\right)$ in decibels) increases as the value of $\omega_{H}$ becomes smaller. For the same value of $\omega_{H}$, the lower bound increases as the tracking error $\bar{u}$ decreases. This conclusion is similar to the results in the homogeneous case.

\section{CONCLUSION AND FUTURE WORK}

In this paper, we have studied the problem of string instability in synchronized harmonic oscillator systems. By using a new integral relation that must be satisfied by the complementary sensitivity function, we provided three sufficient conditions for string instability in homogeneous oscillator systems. We also extended our string instability analysis to heterogeneous oscillator systems where the controllers for different oscillators may be tuned differently and each oscillator can communicate with a few neighbors. In future work, we will consider more complex communication topologies other than strings and stochastic time delays. It will be interesting to extend the analysis in this paper to damped oscillator systems and nonlinear oscillator systems. We will also explore different approaches to improve string stability.

\section{APPENDIX: PROOF OF THEOREM 2}

\section{Proof}

We prove this theorem by making the integration through a contour that includes the ORHP and imaginary axis. The contour is shown in Figure A.1. Several indentations are made to avoid the singularities of $\log T(s)$. The integral around the total contour $C=C_{0}+C_{1}+C_{2}+C_{3}+C_{4}+C_{5}$ is zero. The integral along the imaginary axis, $C_{0}$, satisfies

$$
\begin{aligned}
\lim _{\epsilon \rightarrow 0} \int_{\delta \rightarrow 0} \log [T(s)] \frac{-s^{2}+\alpha^{2}}{\left(s^{2}+\alpha^{2}\right)^{2}} d s & =j \int_{-\infty}^{\infty} \log |T(j \omega)| \frac{\omega^{2}+\alpha^{2}}{\left(\omega^{2}-\alpha^{2}\right)^{2}} d \omega \\
& -\int_{-\infty}^{\infty} \arg [T(j \omega)] \frac{\omega^{2}+\alpha^{2}}{\left(\omega^{2}-\alpha^{2}\right)^{2}} d \omega \\
& =2 j \int_{0}^{\infty} \log |T(j \omega)| \frac{\omega^{2}+\alpha^{2}}{\left(\omega^{2}-\alpha^{2}\right)^{2}} d \omega .
\end{aligned}
$$

The curve $C_{1}$ is a semicircle, which has infinity radius in the ORHP. Hence, $s=R e^{j \theta}$ and $d s=j R e^{j \theta} d \theta$. Then the contribution of this integral $C_{1}$ can be evaluated as 


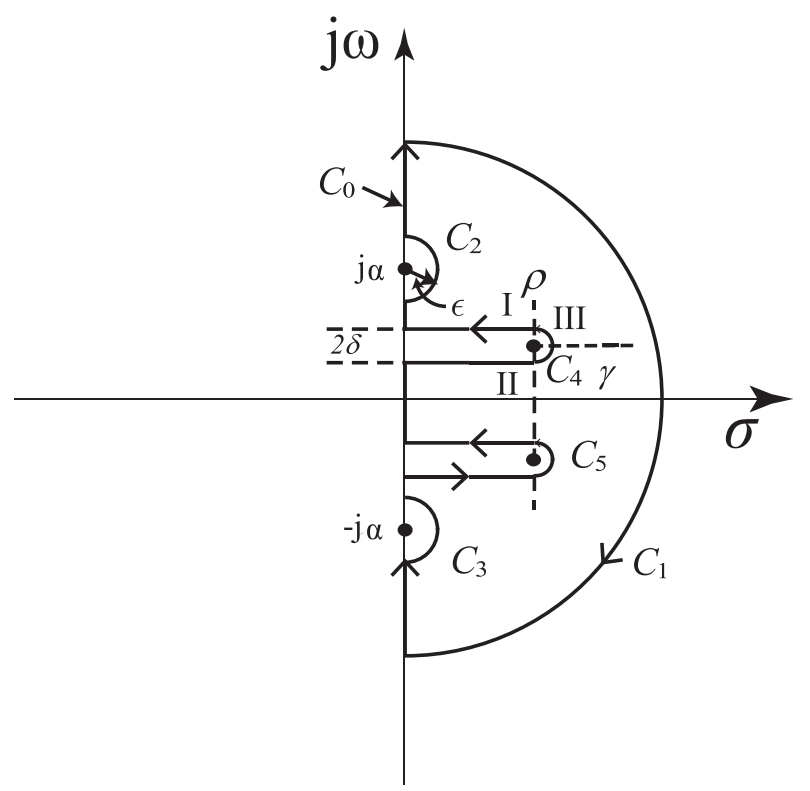

Figure A.1. Contour for complex s-plane used to prove Theorem 2.

$$
\lim _{R \rightarrow \infty} \int_{C_{1}} \log [T(s)] \frac{-s^{2}+\alpha^{2}}{\left(s^{2}+\alpha^{2}\right)^{2}} d s=-j \pi \tau
$$

The integration for $C_{2}$ can be calculated as follows. The radius of the semicircle is $\epsilon$. Hence, $s=j \alpha+\epsilon e^{j \theta},-\frac{\pi}{2} \leqslant \theta \leqslant \frac{\pi}{2}$ and $d s=j \epsilon e^{j \theta} d \theta$. Then

$$
\begin{aligned}
\lim _{\epsilon \rightarrow 0} \int_{C_{2}} \log [T(s)] \frac{-s^{2}+\alpha^{2}}{\left(s^{2}+\alpha^{2}\right)^{2}} d s & =j \int_{-\frac{\pi}{2}}^{\frac{\pi}{2}} \lim _{\epsilon \rightarrow 0} \log \left[T\left(j \alpha+\epsilon e^{j \theta}\right)\right] \frac{1}{-2 \epsilon e^{j \theta}} d \theta \\
& =-j \frac{\pi}{2} \lim _{s \rightarrow j \alpha} \frac{d T(s)}{d s} .
\end{aligned}
$$

Following a similar strategy to calculate the integration for $C_{3}$, we have

$$
\lim _{\epsilon \rightarrow 0} \int_{C_{3}} \log [T(s)] \frac{-s^{2}+\alpha^{2}}{\left(s^{2}+\alpha^{2}\right)^{2}} d s=-j \frac{\pi}{2} \lim _{s \rightarrow-j \alpha} \frac{d T(s)}{d s} .
$$

Furthermore, considering the reflection principal of the analytic function, we obtain

$$
\lim _{\epsilon \rightarrow 0} \int_{C_{2}+C_{3}} \log |T(s)| \frac{-s^{2}+\alpha^{2}}{\left(s^{2}+\alpha^{2}\right)^{2}} d s=-j \pi \operatorname{Re}\left[\lim _{s \rightarrow j \alpha} \frac{d T(s)}{d s}\right] .
$$

The contribution of integrals for $C_{4}$ and $C_{5}$ can be calculated as follows. We first assume that the nonminimum phase zeros are $\rho \pm j \gamma$. First, we need to rewrite $T(s)$ as

$$
T(s)=(s-\rho-j \gamma)(s-\rho+j \gamma) \bar{T}(s) .
$$

Further, we have 


$$
\begin{aligned}
\int_{C_{4}, C_{5}} \log [T(s)] \frac{-s^{2}+\alpha^{2}}{\left(s^{2}+\alpha^{2}\right)^{2}} d s= & \int_{C_{4}, C_{5}} \log (s-\rho-j \gamma) \frac{-s^{2}+\alpha^{2}}{\left(s^{2}+\alpha^{2}\right)^{2}} d s \\
& +\int_{C_{4}, C_{5}} \log (s-\rho+j \gamma) \frac{-s^{2}+\alpha^{2}}{\left(s^{2}+\alpha^{2}\right)^{2}} d s \\
& +\int_{C_{4}, C_{5}} \log \bar{T}(s) \frac{-s^{2}+\alpha^{2}}{\left(s^{2}+\alpha^{2}\right)^{2}} d s .
\end{aligned}
$$

The third term in the right-hand side of (A.5) is zero because the integrand is analytic inside and on the semicircle $C_{4}$ and $C_{5}$. Hence, we focus on the integration of the first and second terms. Consider the line part I, where $s=x+j(\gamma+\delta), 0 \leqslant x \leqslant \rho$. Then $d s=d x$.

$$
\begin{aligned}
\int_{I} \log (s-\rho-j \gamma) \frac{-s^{2}+\alpha^{2}}{\left(s^{2}+\alpha^{2}\right)^{2}} d s= & \int_{\rho}^{0} \log (x-\rho+j \delta) \frac{-(x+j \gamma+j \delta)^{2}+\alpha^{2}}{\left[(x+j \gamma+j \delta)^{2}+\alpha^{2}\right]^{2}} d x \\
= & \int_{\rho}^{0} \ln \sqrt{(\rho-x)^{2}+\delta^{2}} \frac{-(x+j \gamma+j \delta)^{2}+\alpha^{2}}{\left[(x+j \gamma+j \delta)^{2}+\alpha^{2}\right]^{2}} d x \\
& +j \int_{\rho}^{0}\left[\pi+\arctan \left(\frac{\delta}{x-\rho}\right)\right] \frac{-(x+j \gamma+j \delta)^{2}+\alpha^{2}}{\left[(x+j \gamma+j \delta)^{2}+\alpha^{2}\right]^{2}} d x .
\end{aligned}
$$

Following a similar strategy, the integration for II can be calculated as

$$
\begin{aligned}
\int_{I I} \log (s-\rho-j \gamma) \frac{-s^{2}+\alpha^{2}}{\left(s^{2}+\alpha^{2}\right)^{2}} d s= & \int_{0}^{\rho} \ln \sqrt{(\rho-x)^{2}+\delta^{2}} \frac{-(x+j \gamma-j \delta)^{2}+\alpha^{2}}{\left[(x+j \gamma-j \delta)^{2}+\alpha^{2}\right]^{2}} d x \\
& +j \int_{0}^{\rho}\left[-\pi+\arctan \left(\frac{-\delta}{x-\rho}\right)\right] \frac{-(x+j \gamma-j \delta)^{2}+\alpha^{2}}{\left[(x+j \gamma-j \delta)^{2}+\alpha^{2}\right]^{2}} d x .
\end{aligned}
$$

Now, consider the integration for III. Here, $s=\rho+j \gamma+\delta e^{j \theta},-\frac{\pi}{2} \leqslant \theta \leqslant \frac{\pi}{2}$, and $d s=j \delta e^{j \theta} d \theta$.

$$
\begin{aligned}
\lim _{\delta \rightarrow 0} \int_{I I I} \log (s-\rho-j \gamma) \frac{-s^{2}+\alpha^{2}}{\left(s^{2}+\alpha^{2}\right)^{2}} d s & =\lim _{\delta \rightarrow 0} \int_{-\frac{\pi}{2}}^{\frac{\pi}{2}} \log \left(\delta e^{j \theta}\right) \frac{-\left(\rho+j \gamma+\delta e^{j \theta}\right)^{2}+\alpha^{2}}{\left[\left(\rho+j \gamma+\delta e^{j \theta}\right)^{2}+\alpha^{2}\right]^{2}} j \delta e^{j \theta} d \theta \\
& =\lim _{\delta \rightarrow 0} \int_{-\frac{\pi}{2}}^{\frac{\pi}{2}}(\log \delta+j \theta) \frac{-(\rho+j \gamma)^{2}+\alpha^{2}}{\left[(\rho+j \gamma)^{2}+\alpha^{2}\right]^{2}} j \delta e^{j \theta} d \theta \\
& =0 .
\end{aligned}
$$

Note that the first terms in (A.6) and (A.7) cancel out and the second terms are identical when taking $\delta \rightarrow 0$. Hence, we have

$$
\lim _{\delta \rightarrow 0} \int_{C_{4}} \log (s-\rho-j \gamma) \frac{-s^{2}+\alpha^{2}}{\left(s^{2}+\alpha^{2}\right)^{2}} d s=-2 \pi j\left[\frac{\rho+j \gamma}{(\rho+j \gamma)^{2}+\alpha^{2}}-\frac{j \gamma}{-\gamma^{2}+\alpha^{2}}\right] .
$$

Similarly, the integration about the curves for $\rho-j \gamma$, for example, $C_{5}$, has the following results

$$
\lim _{\delta \rightarrow 0} \int_{C_{5}} \log (s-\rho-j \gamma) \frac{-s^{2}+\alpha^{2}}{\left(s^{2}+\alpha^{2}\right)^{2}} d s=-2 \pi j\left[\frac{\rho-j \gamma}{(\rho-j \gamma)^{2}+\alpha^{2}}-\frac{-j \gamma}{-\gamma^{2}+\alpha^{2}}\right] .
$$

Adding (A.8) and (A.9) yields

$$
\lim _{\delta \rightarrow 0} \int_{C_{4}+C_{5}} \log (s-\rho-j \gamma) \frac{-s^{2}+\alpha^{2}}{\left(s^{2}+\alpha^{2}\right)^{2}} d s=-2 \pi j\left[\frac{\rho-j \gamma}{(\rho-j \gamma)^{2}+\alpha^{2}}+\frac{\rho+j \gamma}{(\rho+j \gamma)^{2}+\alpha^{2}}\right] .
$$


The final result then follows considering (A.1), (A.2), (A.3), and (A.10) and noting that the zeros in Theorem 2 occur in conjugate pairs.

\section{ACKNOWLEDGEMENTS}

This research was supported by the National Science Foundation under Grant CNS-1035271 and by the Science Foundation of Ireland under Grant 07/IN.1/I1838.

\section{REFERENCES}

1. Olfati-Saber R, Fax JA, Murray RM. Consensus and cooperation in networked multi-agent systems. Proceedings of the IEEE 2007; 95(1):215-223.

2. Ren W, Beard RW, Atkins EM. Information consensus in multivehicle cooperative control. IEEE Control Systems Magazine 2007; 27(2):71-82.

3. Zhang Y, Yang Y, Zhao Y. Finite-time consensus tracking for harmonic oscillators using both state feedback control and output feedback control. International Journal of Robust and Nonlinear Control 2013; 23(8):878-893.

4. Strogatz SH. Exploring complex networks. Nature 2001; 410(6825):268-276.

5. Chopra N, Spong MW. On exponential synchronization of kuramoto oscillators. IEEE Transactions on Automatic Control 2009; 54(2):353-357.

6. Dörfler F, Chertkov M, Bullo F. Synchronization in complex oscillator networks and smart grids. Proceedings of the National Academy of Sciences 2013; 110(6):2005-2010.

7. Levine WS, Athans M. On the optimal error regulation of a string of moving vehicles. IEEE Transactions on Automatic Control 1966; 11(3):355-361.

8. Peppard LE. String stability of relative-motion PID vehicle control systems. IEEE Transactions on Automatic Control 1974; 19(5):579-581.

9. Swaroop D, Hedrick JK. String stability of interconnected systems. IEEE Transactions on Automatic Control 1996; 41(3):349-357.

10. Seiler P, Pant A, Hedrick K. Disturbance propagation in vehicle strings. IEEE Transactions on Automatic Control 2004; 49(10):1835-1841.

11. Middleton RH, Braslavsky JH. String instability in classes of linear time invariant formation control with limited communication range. IEEE Transactions on Automatic Control 2010; 55(7):1519-1530.

12. Naus GJL, Vugts RPA, Ploeg J, Van de Molengraft MJG, Steinbuch M. String-stable cacc design and experimental validation: a frequency-domain approach. IEEE Transactions on Vehicular Technology 2010; 59(9):4268-4279.

13. Lin F, Fardad M, Jovanovic MR. Optimal control of vehicular formations with nearest neighbor interactions. IEEE Transactions on Automatic Control 2012; 57(9):2203-2218.

14. Seron MM, Braslavsky JH, Goodwin GC. Fundamental Limitations in Filtering and Control. Springer-Verlag New York, Inc.: London, UK, 1997.

15. Toda M. Vibration of a chain with nonlinear interaction. Journal of the Physical Society of Japan 1967; 22(2):431-436.

16. Berman GP, Izrailev FM. The Fermi-Pasta-Ulam problem: fifty years of progress. Chaos: An Interdisciplinary Journal of Nonlinear Science 2005; 15(1):015 101

17. Ren W. Synchronization of coupled harmonic oscillators with local interaction. Automatica 2008; 44(12):3195-3200

18. Yu B, Freudenberg JS, Gillespie RB, Middleton RH. String instability in coupled harmonic oscillator systems. Proceedings of the 50th IEEE Conference on Decision and Control, Orlando, FL, 2011; 6224-6229.

19. Yu B, Freudenberg JS, Gillespie RB. String instability analysis of heterogeneous coupled oscillator systems. Proceedings of the 2012 American Control Conference, Montreal, QC, 2012; 6638-6643.

20. Brown JW, Churchill RV. Complex Variables and Application. McGraw-Hill Science/Engineering/Math: New York, USA, 2008

21. Conway JB. Functions of One Complex Variable (2nd edn). Springer-Verlag: New York, USA, 1978.

22. Barooah P, Mehta PG, Hespanha JP. Mistuning-based control design to improve closed-loop stability margin of vehicular platoons. IEEE Transactions on Automatic Control 2009; 54(9):2100-2113.

23. Davison EJ. The robust control of a servomechanism problem for linear time-invariant multivariable systems. IEEE Transactions on Automatic Control 1976; 21(1):25-34 